\title{
Forced Kramers escape in single-molecule pulling experiments
}

\author{
Yu-Jane Sheng \\ Department of Chemical Engineering, National Taiwan University, Taipei, Taiwan 106, Republic of China \\ Shaoyi Jiang \\ Department of Chemical Engineering, University of Washington, Seattle, Washington 98195 \\ Heng-Kwong Tsao ${ }^{\text {a) }}$ \\ Department of Chemical and Materials Engineering, National Central University, Jhongli, Taiwan 320, \\ Republic of China
}

(Received 27 June 2005; accepted 4 August 2005; published online 8 September 2005)

\begin{abstract}
The pulling-induced rupture of noncovalent bonds is studied by the overdamped Kramers theory with full account of a time-varying barrier. Mechanic pulling reduces the energy barrier and leads to loading-rate dependence of the rupture force $F_{u}\left(\mathcal{F}_{t}\right)$. Tested against Langevin dynamics, four distinct regimes are identified, including kinetic dominant, weak pulling, strong pulling, and mechanic pulling dominant. Asymptotic analyses show that $F_{u} \sim \ln \mathcal{F}_{t}$ in weak pulling regime and becomes $1-\left(F_{u} / F_{c}\right) \sim\left[\ln \left(\mathcal{F}_{t}\right) / E_{b}\right]^{2 / 3}$ in strong pulling regime. Kinetic informations such as activation energy $E_{b}$ and critical force $F_{c}$ were extracted from pulling experiments for biotin-streptavidin complex. (C) 2005 American Institute of Physics. [DOI: 10.1063/1.2046632]
\end{abstract}

The physical nature of noncovalent biomolecular bonds has been experimentally explored at the single-molecule level. ${ }^{1,2}$ In these experiments such as ligand-receptor dissociation and protein unfolding, an externally pulling force (in the piconewton range) is exerted on an anchored molecular complex. There are various ultrasensitive force-probing techniques including biomembrane force probe (BFP), atomic force microscope (AFM), and optical tweezers. In the BFP and AFM experiments, the force is slowly increased until bond rupture occurs. Typically, the pulling spring is moved away from the anchored molecule at a constant velocity. After sufficiently long time, it leads to a constant rate of force applying to the noncovalent bonds. In contrast to equilibrium binding properties, the bond-rupture force, employed in depicting bond strength, is not constant but instead dependent upon the loading rate $\mathcal{F}_{t}$.

The long natural lifetime of a ligand-receptor complex indicates that the thermally activated escape (unbinding at zero force) must cross a large energy barrier. Subjected to forces, the kinetic escape across the barrier is assisted mechanically and the escape time scale is reduced. A given bond will break eventually under any level of pulling if applied over a sufficient time period. When the ramping rate is very slow, the time is long enough so that the rupture occurs mainly by thermal activation and low rupture forces are resulted. On the other hand, if the ramping rate is very fast then no time is available for accumulated thermal actions and the rupture force approaches maximum intrinsic binding force.

In an effort to interpret the force-probing experiments in terms of potential energy surface and to develop insights into the force-induced dissociation, molecular-dynamics (MD) studies have been undertaken. ${ }^{3,4}$ Owing to computational

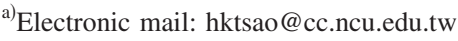

limits, MD simulations operate at the nanosecond time scale, which is opposed to the time scale of the dynamic force spectroscopy as well as spontaneous dissociation (millisecond to second). Therefore, large loading rates, several orders of magnitude greater than experimental rates, are employed to achieve rupture within the time available. Nonetheless, such simulations overcome all energy barriers mechanically and frictional forces play an important role.

An alternative approach to explore the unbinding process is the Kramers rate theory. In the seminal paper, ${ }^{5}$ Evans and Ritchie showed that time-ramped pulling lowers the energy barrier and leads to loading-rate dependence of the rupture force. On the basis of the Kramers rate theory, they found that the dynamic bond strength increased linearly with the logarithm of the loading rate. To introduce the effect of pulling on the dissociation rate constant, they adopted Bell's approximation which is valid only for weak pulling. To interpret distinct regimes shown in the force spectroscopy data, multiple barriers were thus invoked. Unfortunately, recent overdamped Langevin dynamics illustrated that the logarithmic law is inadequate. ${ }^{6,7}$

In this Communication, we correctly account for the time-varying barrier and preexponential factor in overdamped Kramers theory. The results are examined by the Langevin dynamics. Our goal is to find the scaling behavior of the rupture force, which allows one to extract information associated with intrinsic kinetic escape (spontaneous dissociation) from the single-molecule pulling experiments. Four distinct regimes are observed for a single barrier, including kinetic escape dominant, weak pulling, strong pulling, and mechanic pulling dominant.

The unbinding process of the bound complex along the reaction coordinate $x$ is equivalent to the escape of an overdamped particle from a kinetic trap $U(x)$ under the action of 
a time-dependent force $F(t)$. The Langevin equation for this particle of mass $M$ reads $^{8}$

$$
M \gamma \frac{d x}{d t}=-\frac{d}{d x}[U(x)-F(t) x]+\xi(t),
$$

where the effect of thermal fluctuations is represented by a random force and obeys the correlation $\left\langle\xi(t) \xi\left(t^{\prime}\right)\right\rangle$ $=2 M \gamma k_{B} T \delta\left(t-t^{\prime}\right) . M \gamma$ denotes the friction coefficient associated with the Brownian motion on the free-energy surface and relates to the diffusivity $D$ by $M \gamma=k_{B} T / D$. The freeenergy surface is characterized by the distance from the metastable well to the saddle point $a$ and the energy barrier $E_{a}=U(a)-U(0)$. In addition, the maximum slope of the trapping potential denotes the critical force $F_{c}$, which plays an important role when an external force is applied. When pulling is absent, the bound complex dissociates eventually. This thermally activated escape characterizes the kinetic limit. In contrast, when $F>F_{c}$, the dissociation is dominated by mechanic pulling. This is referred to as the mechanic limit. Under fixed pulling $F<F_{c}$, the free-energy surface is altered. Because the energy barrier is lowered, the mean first passage time of the forced escape from a kinetic trap is reduced with increasing $F$. When the applied pulling is ramped up with time, the interplay between kinetic escape and mechanic pulling leads to ramping-rate dependent behavior. The timeramped force at rupture, corresponding to $F_{u}=F(x=a)$, depends on the lifetime that is stochastic in nature due to kinetic escape. Although the rupture force is not an intrinsic property of the bound complex, the goal is to extract properties of the free-energy surface from pulling experiments.

The kinetics of bond rupture governed by Eq. (1) can be depicted by a phenomenological formalism. The survival probability $P_{s}(t)$ satisfies the first-order rate equation with a time-dependent rate constant $k(t)$,

$$
\frac{d P_{s}}{d t}=-k(t) P_{s}(t) .
$$

Therefore, $P_{s}(t)=\exp \left[-\int_{0}^{t} k\left(t^{\prime}\right) d t^{\prime}\right]$. The rate constant can be calculated by Kramers theory,

$$
k=\nu \exp \left(-\beta E_{b}\right) \quad \text { with } \nu=\frac{\omega_{-} \omega_{+}}{2 \pi \gamma} .
$$

Here $\omega_{ \pm}=\left[U^{\prime \prime}\left(x_{ \pm}\right) / M\right]^{1 / 2}$ are the oscillation frequencies at the well $\left(x_{-}\right)$and the saddle point $\left(x_{+}\right)$, and the activation energy is $E_{b} \gg k_{B} T$. When the pulling is applied, the effective trapped potential in Eq. (1) becomes $V(x)=U(x)-F(t) x$. Thus, the well and saddle point change with the time-ramped force and are determined by $U^{\prime}\left[x_{ \pm}(t)\right]=F(t)$ due to $\partial V / \partial x$ $=0$. The barrier height then becomes $E_{b}(t)=V\left[x_{+}(t)\right]$ $-V\left[x_{-}(t)\right]$ and the preexponential factor $\nu\left[x_{ \pm}(t)\right]$. Since the probability distribution of force $F$ at rupture is related to the probability distribution of lifetimes by $p(F) d F$ $=k\left(t^{*}\right) P_{s}\left(t^{*}\right) d t^{*}$, the mean rupture force is given by

$$
\left\langle F_{u}\right\rangle=\int_{0}^{\infty} F(t) k(t) \exp \left[-\int_{0}^{t} k\left(t^{\prime}\right) d t^{\prime}\right] d t .
$$

Note that $p(F) \rightarrow 0$ as $F \rightarrow F_{c}$.
When the loading rate $\left(\mathcal{F}_{t}=d F / d t\right)$ is slow enough, the free-energy landscape is slightly perturbed and it is reasonable to adopt the adiabatic approximation analogous to what is done in quantum mechanics. For any known potential $U(x)$, the integration in Eq. (4) can be performed numerically to obtain the rupture force $\left\langle F_{u}\right\rangle$. Nonetheless, it would be difficult to extract information from experimental results by Eq. (4). Two analytical expressions can be obtained in the asymptotic limits $F_{u} / F_{c} \ll 1$ and $1-F_{u} / F_{c} \ll 1$.

(i) When $F_{u} \ll F_{c}$, the well and saddle point are slightly perturbed from the force-free positions $x_{ \pm}(t) \cong x_{ \pm}^{0}$ $+\left[F(t) / U^{\prime \prime}\left(x_{ \pm}^{0}\right)\right]$. Here $x_{ \pm}^{0}$ denote the positions with $F$ $=0$ and $x_{+}^{0}-x_{-}^{0}=a$. As $F\left[1 / U^{\prime \prime}\left(x_{-}^{0}\right)-1 / U^{\prime \prime}\left(x_{+}^{0}\right)\right] / a \ll 1$, one has

$E_{b} \cong E_{a}-F(t) a$ and $\omega_{ \pm}=\omega_{ \pm}^{0}$.

This result is simply the well-known Bell expression., ${ }^{5,9}$ Using Eq. (5) in Eq. (3) and carrying out the integration in Eq. (4) yields

$\beta a\left\langle F_{u}\right\rangle=\exp \left(\frac{k_{0}}{\beta a \mathcal{F}_{t}}\right) E_{1}\left(\frac{k_{0}}{\beta a \mathcal{F}_{t}}\right)$,

where $E_{1}(x)$ is the exponential integral and $k_{0}$ denotes the rate constant in the absence of pulling $k_{0}=k(F=0)$. Dependent on the loading rate, two regimes can be identified. When $\mathcal{F}_{t} \ll k_{0} / \beta a$, one has

$\left\langle F_{u}\right\rangle \simeq \mathcal{F}_{t} / k_{0}$.

On the contrary, as $\mathcal{F}_{t} \gg k_{0} / \beta a$, logarithmic law is obtained,

$\left\langle F_{u}\right\rangle \cong \frac{1}{\beta a}\left[\ln \left(\frac{\beta a \mathcal{F}_{t}}{k_{0}}\right)-\lambda\right]$,

where $\lambda=0.5772 \ldots$ is the Euler constant. In order to satisfy the assumption, the valid range of the logarithmic law regime is $e^{\beta E_{a} \gg \beta a \mathcal{F}_{t} / k_{0} \gg 1}$.

(ii) When the loading rate is sufficiently fast, the kinetic escape takes place primarily as $F \rightarrow F_{c}$, at which the effective barrier disappears completely. Consequently, it is a good approximation to assume the bond strength as $U^{\prime}(x)=F_{c}\left[1-\left(x-x_{c}\right)^{2} / b^{2}\right]$. It is obtained by Taylor expansion around the critical force. As shown in Fig. 1, $2 b=\left[-8 F_{c} / U^{\prime \prime \prime}\left(x_{c}\right)\right]^{1 / 2}$ denotes the interaction range associated with the approximated cubic potential. The activation energy and oscillation frequencies are then related to the force by

$E_{b}=E_{a}^{*}\left[1-\frac{F(t)}{F_{c}}\right]^{3 / 2}$ and $\omega_{ \pm}=\omega_{ \pm}^{*}\left[1-\frac{F(t)}{F_{c}}\right]^{1 / 4}$,

where $E_{a}^{*}$ and $\omega_{ \pm}^{*}$ are associated with the cubic potential. Performing asymptotic integration for $\beta E_{b} \gg 1$ (Ref. 10) gives 


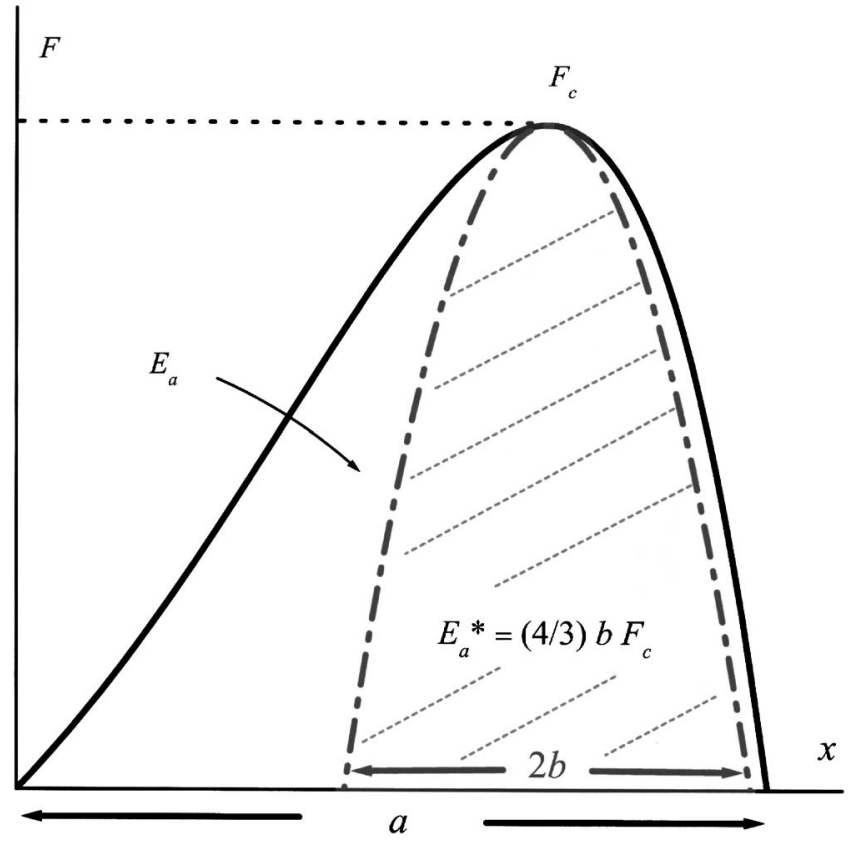

FIG. 1. Schematic representations of the kinetic trap and the approximated trap associated with cubic potential at strong pulling regime.

$$
\begin{aligned}
\left\langle F_{u}\right\rangle \cong & F_{c}\left[1-\left(-\frac{\ln 2 \beta b \nu_{*}^{-1} \mathcal{F}_{t}}{\beta E_{a}^{*}}\right)^{2 / 3}\right. \\
& \left.\times\left(1-\frac{2 \lambda}{3 \ln 2 \beta b \nu_{*}^{-1} \mathcal{F}_{t}}\right)\right] .
\end{aligned}
$$

Note that $\mathcal{F}_{t}<\nu_{*} / 2 \beta b$ and $E_{a}^{*}=\frac{4}{3} F_{c} b$ for the cubic potential.

In scaling analysis the different loading-rate-dependent regimes associated with forced escape from a kinetic trap can be identified by the competition between the two characteristic time scales: the kinetic escape time $\tau_{k}$ and the mechanic pulling time $\tau_{m}$. The former is inversely proportional to the rate constant, $\tau_{k}(F) \sim k^{-1}(F)$, which varies with the external force. On the other hand, for steadily increasing loading, the escape time determined by mechanic pulling is $\tau_{m}(F)$ $\sim F / \mathcal{F}_{t}$. As demonstrated in Fig. 2, four distinct regimes can be obtained as follows: (I) Kinetic escape dominant regime. In the absence of pulling, $\tau_{k}(F=0)=\tau_{k}^{0}$. When $\tau_{k}^{0} \ll \tau_{m}\left(F_{c}\right)$, the kinetic escape occurs far before mechanic pulling reaches the critical force and the escape is mainly governed by thermal activation. The work done by the pulling force is negligible compared to the energy barrier, i.e., $E_{a} \gg \mathcal{F}_{t} \tau_{k}^{0} a$ or $F_{u} / F_{c} \rightarrow 0$. Note that $E_{a} \sim F_{c} a$. As a result, the rupture force is simply the loading rate multiplied by the kinetic escape time, $F_{u} \sim \mathcal{F}_{t} \tau_{k}^{0}=\mathcal{F}_{t} / k$. This linear-loading rate-dependent behavior, as shown in Eq. (7), takes place at extremely slow ramping rate and is difficult to be observed in the present pulling apparatus. (II) Weak pulling regime. The energy barrier is weakly altered by the external pulling and hence $\tau_{k}(F) \approx \tau_{k}^{0} \exp (-\beta a F)$. The condition $\tau_{k}\left(F_{u}\right) \sim \tau_{m}\left(F_{u}\right)$ yields $\beta a F_{u} \sim \ln \beta a \tau_{k}^{0} \mathcal{F}_{t}$ if $\beta E_{a} \gg \beta a F_{u} \gg \ln \beta a F_{u}$. This logarithmic loading rate-dependent behavior, as shown in Eq. (8), occurs at sufficiently slow ramping rate. (III) Strong pulling regime.

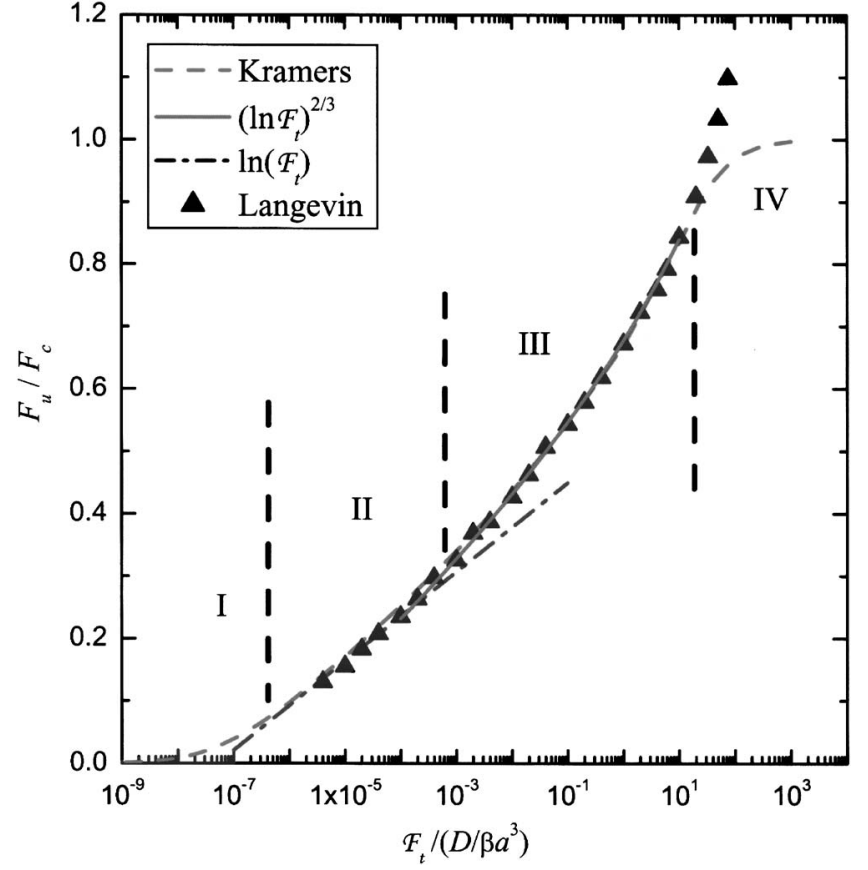

FIG. 2. The variation of the mean rupture force with the loading rate for $\beta E_{a}=20$. The four regimes are identified.

The energy barrier is significantly altered and $\tau_{k}(F)$ $\approx \nu_{*}^{-1} \beta E_{a}^{*} \exp \left[\beta E_{a}^{*}\left(1-F / F_{c}\right)^{3 / 2}\right]$. The condition $\tau_{k} \sim \tau_{m}$ gives $F_{u} / F_{c} \sim 1-\left[-\ln \left(2 \beta b \nu_{*}^{-1} \mathcal{F}_{t}\right) / \beta E_{a}^{*}\right]^{2 / 3}$ if $F_{u} \rightarrow F_{c}$. This outcome agrees with Eq. (10). (IV) Mechanic pulling dominant regime. When the loading rate is sufficiently fast, the pulling reaches the critical force before the kinetic escape occurs, i.e., $\tau_{m}=F_{c} / \mathcal{F}_{t} \ll \tau_{k, \min }$. Consequently, the escape is governed by the mechanic pulling. The maximum rate constant estimated by $\partial k / \partial F=0$ gives $\tau_{k, \min }=k_{\max } \sim \nu_{*}^{-1}\left(\beta E_{a}^{*}\right)^{1 / 3}$. Hence, the valid range of this regime is $\beta b \nu_{*}^{-1} \mathcal{F}_{t} \gg\left(\beta E_{a}^{*}\right)^{2 / 3}$. Note that the assumption of an adiabatic trap is justified if $\tau_{m}$ $\gg \tau_{r}$ which is the molecular relaxation time. Moreover, friction becomes significant because $(M \gamma d x / d t) / F_{c} \gg\left(\beta E_{a}\right)^{-1 / 3}$ in this regime. In addition to time scales, another factor to distinguish those regimes is the ratio of the work done by mechanic pulling to the activation energy. That is, regime (I) $F_{u} a / E_{a} \rightarrow 0$, (II) $F_{u} a / E_{a} \ll 1$, (III) $F_{u} b / E_{a} y 1$, and (IV) $F_{u} b / E_{a}>1$.

In order to examine the validity of Eq. (4) and the applicability of Eqs. (8) and (10), we perform the forced escape simulations governed by the Langevin equation (1) for an arbitrarily chosen potential, $U(x)=E_{a} x^{2}\left(4 x^{3}-5 x^{2}-10 x\right.$ $+15) / 4$ with $x$ scaled by $a$. For this potential with $\beta E_{a}=20$, $F_{c}=32.2 \mathrm{kT} / a$ at $x_{c}=0.422$ and $2 b=0.872$. The simulation results of mean rupture forces are shown in Fig. 2 with loading rates spanned over eight orders of magnitude. They can be well represented by Eq. (4) in regimes (II) and (III). This consequence confirms the success of the Kramers theory with a time-varying barrier. In regime (IV), the friction in Eq. (1) becomes substantial and $F_{u}$ must overcome both $F_{c}$ and $M \gamma d x / d t$ to result in escape. However, the Kramers theory does not take this effect into account and $F_{u} \leqslant F_{c}$. Figure 2 also justifies the two asymptotic expressions, Eqs. (8) and (10) and confirms the existence of the weak and 


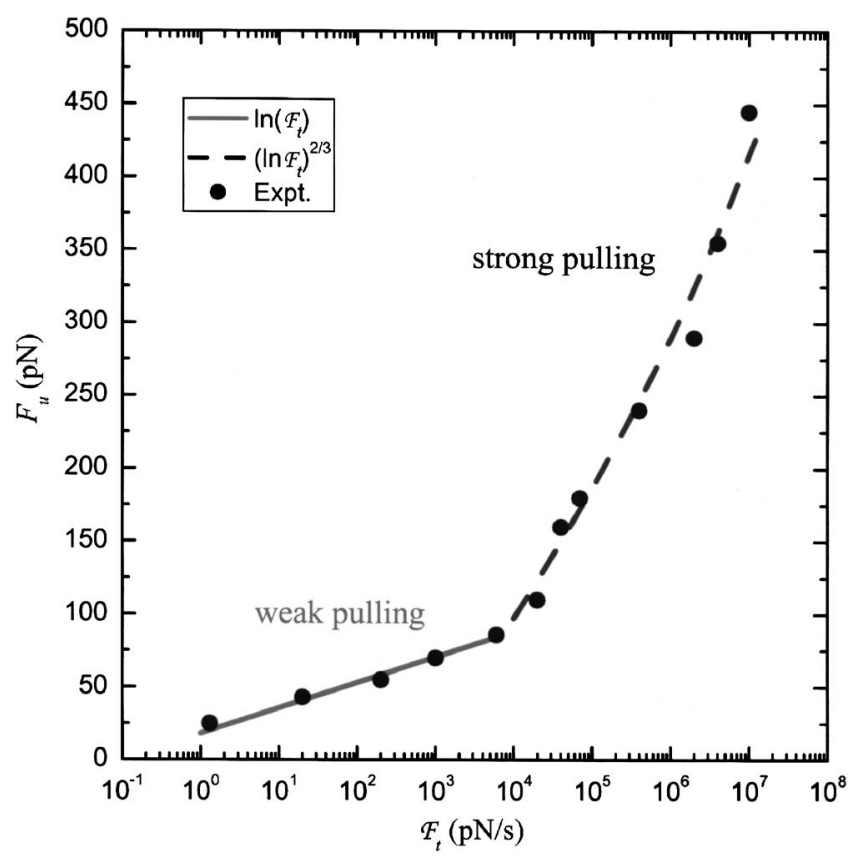

FIG. 3. Comparison between the experimental data of AFM and $\mathrm{BFP}^{2,10}$ and Eqs. (8) and (10). The kinetic information such as critical force and activation energy can therefore be extracted.

strong pulling regimes. As a result, one can use these two analytical expressions to extract kinetic information from pulling experiments.

We consider the mean rupture forces obtained by the recent AFM and BFP measurements for unbinding biotinstreptavidin complex. ${ }^{2,11}$ The combined spectrum were often regarded as two linear regimes and two energy barriers along the unbinding pathway are invoked to explain such behavior. For each barrier a characteristic force $f_{\alpha}$ is related to the projected bond displacement $x_{\alpha}$ along the reaction coordinate, i.e., $f_{\alpha}=k_{B} T / x_{\alpha}$. Evidently, $f_{\alpha} \ll F_{c}$ because $f_{\alpha} x_{\alpha}$ denotes the thermal energy only. The intrinsic rate constant $k_{0}$ was determined from the linear extrapolation to zero unbinding force $\mathcal{F}_{t}^{0}\left(F_{u}=0\right)$ by $\left(k_{0}\right)_{\alpha}=\left(\mathcal{F}_{t}^{0}\right)_{\alpha / f \alpha}$, which is about $O\left(10^{-2}-10\right) \mathrm{s}^{-1}$. Our analysis shows that the experimentally observed upturn in the $F_{u}-\mathcal{F}_{t}$ plot does not necessarily imply the existence of multiple barriers. ${ }^{2,12}$ A single barrier can display a linear regime (II) and another weakly nonlinear regime (III). Figure 3 shows that the dynamic force spectra can be well represented by the asymptotic expressions: Eqs. (8) and (10). Fitting experimental data at low loading rates to the logarithmic law, Eq. (8), gives the interaction range at weak pulling regime $a=5.4 \AA$ and the intrinsic rate constant $k_{0}=6.92 \times 10^{-3} \mathrm{~s}^{-1}$. On the other hand, fitting $F_{u}$ at high loading rates to Eq. (10) yields the critical force $F_{c}$ $=543.3 \mathrm{pN}$, the apparent activation energy $\beta E_{a}^{*}=10.83$, and $2 \beta b \nu_{*}^{-1}=5.68 \times 10^{-8} \mathrm{~s} / \mathrm{pN}$. Since $\frac{3}{2} \beta E_{a}^{*}=2 b F_{c}$ for cubic potential, one obtains the interaction range at strong pulling regime $2 b=1.23 \AA$ and thereby $\nu_{*}=5.26 \times 10^{5} \mathrm{~s}^{-1}$. The dif- fusivity (or friction coefficient) on the free-energy surface can also be estimated from $\nu_{*}=(3 / \pi) \beta E_{a}^{*} /\left(2 b^{2} / D\right)$, i.e., $D$ $=6.16 \times 10^{-15} \mathrm{~m}^{2} / \mathrm{s}$ and $M \gamma=6.7 \times 10^{-7} \mathrm{~kg} / \mathrm{s}$. Because $M \omega_{-} \omega_{+} \approx\left(F_{c} / b\right)[(a / b)-1]^{-1 / 2}$ is quite close to $M \omega_{-}^{*} \omega_{+}^{*}$ $=2 F_{c} / b$ associated with the apparent cubic potential, it is reasonable to assume $\nu \approx \nu_{*}$. Then the intrinsic activation energy can be estimated from $k_{0}$ as $\beta E_{a} \simeq 18.15$ with the error of $\ln \left(\nu / \nu_{*}\right)$. The calculated parameters of energy landscape are typical for ligand-receptor bonds.

Forced escape form a single barrier trap can be well described by the Kramers theory with full account of a timevarying activation energy. The "adiabatic" assumption inherent in our analysis are justified for slow driving. Note that analytical theories for the time-dependent overdamped Kramers problem are recently developed for periodic driving. They are valid over a wide range of driving strength and driving frequencies. ${ }^{13-15}$ Four distinct regimes in the $F_{u}-\mathcal{F}_{t}$ plot are identified. Our analysis show that dynamic force spectra experiments exhibit weak and strong pulling regimes, which provide kinetic informations about the energy landscape. By fitting experimental data to analytical expressions, the weak pulling regime gives the intrinsic rate constant $k_{0}$ and overall interaction range $a$. The strong pulling regime offers critical force $F_{c}$, apparent activation energy $E_{a}^{*}$, and ratio of strong pulling range to preexponential factor $b \nu_{*}^{-1}$. Further analysis yields the activation energy $E_{a}$ and diffusivity $D$. Our method to extract kinetic information from pulling experiments is simple to implement and should be proved useful in analyzing a variety of experimental systems.

One of the authors (S.J.) gratefully acknowledge the financial support from NSF. Two of the authors (Y.-J.S. and H.-K.T.) thank National Council of Science of Taiwan for financial support. Computing time provided by the National Center for High-Performance Computing of Taiwan is gratefully acknowledged.

${ }^{1}$ E. L. Florin, V. T. Moy, and H. E. Gaub, Science 264, 415 (1994).

${ }^{2}$ R. Merkel, P. Nassoy, A. Leung, K. Ritchie, and E. Evans, Nature (London) 397, 50 (1999).

${ }^{3}$ H. Grubmüller, B. Heymann, and P. Tavan, Science 271, 997 (1996).

${ }^{4}$ S. Izrailev, S. Stepaniants, M. Balsera, Y. Oona, and K. Schulten, Biophys. J. 72, 1568 (1997).

${ }^{5}$ E. Evans and K. Ritchie, Biophys. J. 72, 1541 (1997)

${ }^{6}$ G. Hummer and A. Szabo, Biophys. J. 85, 5 (2003).

${ }^{7}$ O. K. Dudko, A. E. Filippov, J. Klafter, and M. Urbakh, Proc. Natl. Acad. Sci. U.S.A. 100, 11378 (2003).

${ }^{8}$ P. Hänggi, P. Talkner, and M. Borkovec, Rev. Mod. Phys. 62, 251 (1990).

${ }^{9}$ G. I. Bell, Science 200, 618 (1978).

${ }^{10}$ A. Garg, Phys. Rev. B 51, 15592 (1995).

${ }^{11}$ Y.-S. Lo, Y.-J. Zhu, and T. P. Beebe, Jr., Langmuir 17, 3741 (2001).

${ }^{12}$ E. Evans, A. Leung, D. Hammer, and S. Simon, Proc. Natl. Acad. Sci. U.S.A. 98, 3784 (2001).

${ }^{13}$ V. N. Smelyanskiy, M. I. Dykamn, and B. Golding, Phys. Rev. Lett. 82, 3193 (1999).

${ }^{14}$ J. Lehmann, P. Reimann, and P. Hänggi, Phys. Rev. Lett. 84, 1639 (2000); J. Lehmann, P. Reimann, and P. Hänggi, Phys. Rev. E 62, 6282 (2000).

${ }^{15}$ P. Talkner and J. Luczka, Phys. Rev. E 69, 046109 (2004). 